\title{
PAIR-WISE COMPARISON MCDA APPROACH FOR LARGE DMU SETS
}

\author{
Mirosław Dytczak* \\ Opole University of Technology \\ Opole, Poland \\ E-mail: mdytczak@gmail.com \\ Grzegorz Ginda \\ Opole University of Technology \\ Opole, Poland \\ E-mail:gginda@gmail.com
}

\begin{abstract}
AHP/ANP is a recognised multi-attribute decision support method. It is, however, easily and directly applicable rather only in cases of small amount of decision making units (DMUs). AHP/ANP implements some mechanisms which make addressing of larger sets DMUs possible. For example, division of DMUs population into several small groups can help with this regard. It still proves nevertheless cumbersome in the case of considerably large sets of DMUs. A special approach for addressing large sets of DMUs is presented in the paper. It includes several phases. At first, AHP/ANP-tractable number of similar DMUs clusters are obtained. Rankings of DMUs inside clusters and rankings of clusters are delivered then us ing pair-wise AHP/ANP comparisons. Obtained rankings make it possible to obtain the overall ranking of DMUs. Application of a concept of disaggregation and aggregation of DMUs set makes the approach even more powerful. Results of presented analysis confirm its usefulness.
\end{abstract}

Keywords: MCDA, multi-phase approach, numerous DMUs, pair-wise comparison, AHP/ANP, cluster analysis.

\section{Introduction}

Presence of numerous decision alternatives makes decision analysis a challenging task. Nature of contemporary decision making problems makes including both tangibles and intangibles. Prioritisation of considered objects with regard to tangible features is rather straightforward due to direct availability of evaluations. There are consistent methods available which make including of intangibility easy. The methods are, however, at least inconvenient in use in the case of numerous DMUs. The AHP/ANP is not different with this regard.

A two-phase, AHP/ANP-based, approach is presented which allows to rank large sets of unique DMUs to obtain their ranking with regard to intangible features. The approach is capable of dealing with considerably large sets of evaluated decision alternatives. It delivers results which are compatible with object evaluations due to tangible features. Final ranking or clusters of objects can be thus obtained which make attaining at efficient decision possible in the case of numerous DMUs and including of intangibles.

\footnotetext{
${ }^{*}$ Corresponding author
} 


\section{The proposed approach}

\subsection{Structure of the approach}

The approach considers a large set of DMUs. Both tangible and intangible DMU features are included. Local priorities of DMUs with regard to both kinds of features are utilised to obtain the ir ranking and clusters. The approach is divided into two main stages:

1. Local prioritisation of DMUs with regard to tangible and intangible features.

2. Ranking and identification of clusters consisting of the closest DMUs. Different nature of tangible and intangible DMU features results in two separate prioritisation processes. Availability of evaluations of DMUs with regard to tangible features makes prioritisation due to tangibles an easy task. Deriving of priorities with regard to intangibles, however, differs a lot. Application of a separate process is therefore required in their case.

Both processes should deliver compatible local priority descriptions to make the m seamless. Application compatible descriptions makes final aggregation and clustering of DMUs easy. There are several description alternatives available: idealised, normalised and unitarised priorities. They are all non-negative but differ in intervals of derived evaluations, aggregation formulae applicability and suitability for cluster analys is. Idealised priorites are not greater than one, sum of normalised priorities is equal to one and unitarised priorities range from 0 (the least preferable DMU) to 1 (the most preferable DMU). Idealised and normalised priorities allow application of both additive and multiplicative AHP/ANP aggregation models in the case of hierarchical model utilisation. Unitarisation priorities are suitable for cluster analysis application and multiplicative aggregation.

The approach allows to include differences in importance of considered features of DMUs. AHP/ANP hierarchy is applied for derivation of final values of feature importance weights. It is possible to prioritise numerous features using the approach devoted to multiple DMUs which is presented in the next subsection.

General scheme for the approach includes therefore three separate processes which deliver local priorities for:

1. Attributes of DMUs.

2. DMUs with regard to tangible features.

3. DMUs with regard to intangible features.

Different methods can be applied for aggregation of final priorities. Both typical AHP/ANP additive and multiplicative aggregation as well as even several methods in parallel (Dytczak, \& Szklennik, 2011) can be utilised with this regard. Several forms of final ranking can be thus obtained which would make analysis more justified.

Application of cluster analysis and discriminant analysis methods can be utilised for identification of DMU clusters. Available tools are presented in literature (Kolenda, 2006). Likewise in the case of rankings, parallel application of different clustering and discrimination methods would make the analys is even more justified.

General scheme for the approach is presented in Fig.1.

\subsection{Prioritisation of numerous DMUs with regard to intangible features}

Utilisation of AHP/ANP makes including intangibles easy. Its application allows to include not more that nine objects at once as consequence of the well known Miller's rule (Miller, 1956; Saaty, \& Ozdemir, 2003). There is, however, a possibility to divide evaluated objects into several groups of AHP/ANP-tractable cardinality. Each group should contain similar objects with regard to considered feature and one DMU which is common for two different groups (pivot object). Priorities for DMUs are then separately derived inside each identified group and common. Priorities obtained for DMUs inside 
each group are then unified using priorities derived for pivot objects. Proposed approach makes also use of grouping of similar objects. It doesn't apply, however, pivot objects. Avoiding utilisation of pivot objects serves simplification of prioritisation process.

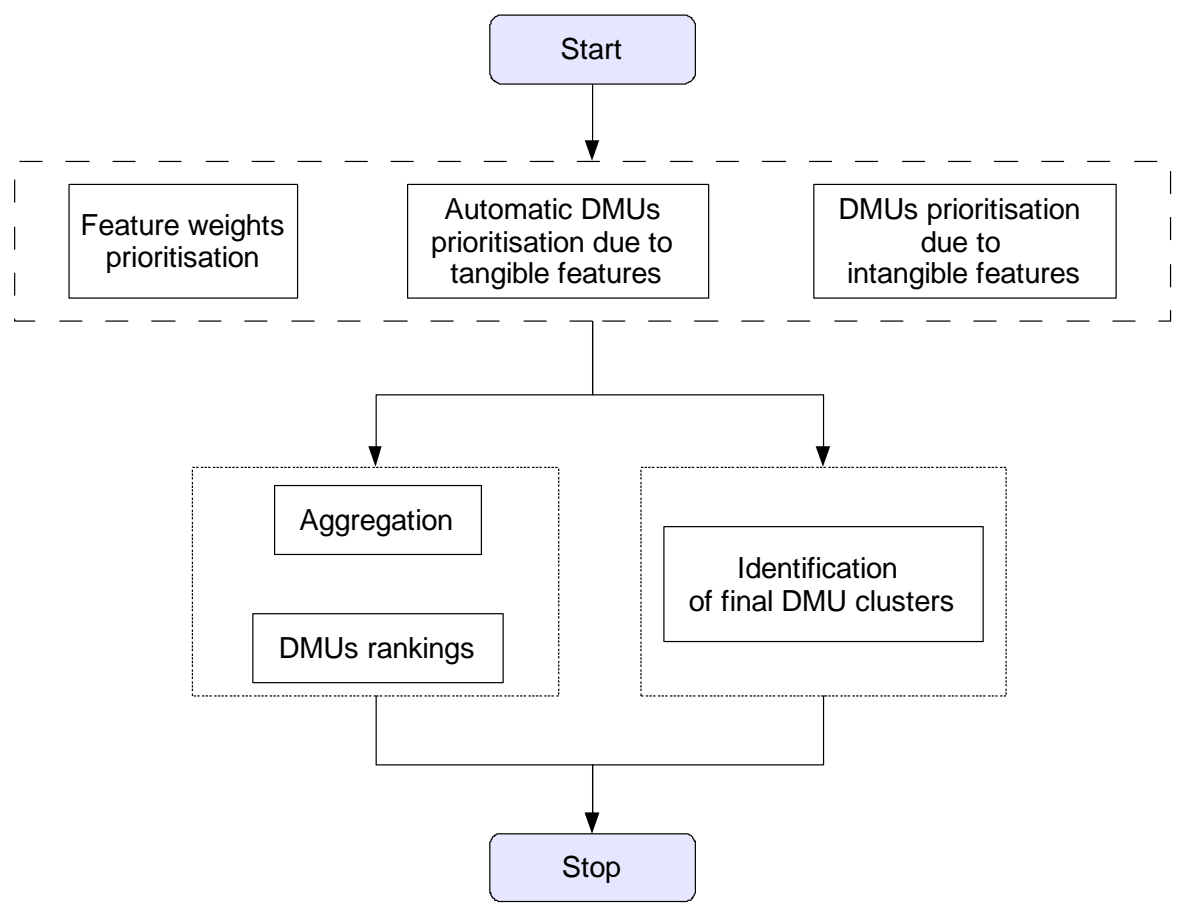

Figure 1. General scheme for the approach

Application of hierarchy of objects - DMU clusters and DMUs comprises the basis of proposed approach. The hierarchy resembles typical AHP/ANP control hierarchy. Its bottom-most level consists of DMUs and intermediate levels are occupied by DMU clusters. The intermediate levels can also include individual DMUs. It isn't, however, desirable due to a hierarchy structure consistency.

AHP/ANP-like prioritisation is applied for deriving level-wise local priorities for objects which occupy a hierarchy level. Each hierarchy level shouldn't therefore contain more than nine objects because of AHP/ANP-tractability issues. Minimal number of object shouldn't be also less than five due to Miller's rule (Miller, 1956; Saaty, \& Ozdemir, 2003). Number of essential hierarchy levels (those which contain clusters and DMUs) is limited too. Up to seven essential levels are usually applied.

Restriction with regard to cardinality of hierarchy levels and DMU cluster thus results in the upper limit of DMUs which is equal to: $9^{7}$ i.e. 4782969 . This is a really huge number as it is obvious that a large group of evaluated objects can contain multiple object with the same level of feature evaluation.

Local priorities are obtained on a usual AHP/ANP level-by-level aggregation of priorities obtained for objects at each level. Normalised level-wise object priorities are applied with this regard. Application of additive aggregation of level priorities gives normalised priorites for DMUs then. A course of a local priority estimation for a DMU 332 (which indirectly belongs to clusters Cluster 122 and Cluster 12) is illustrated in Fig.2.

The approach is open with regard to application of alternative AHP/ANP preference measurement scales and prioritisation methods. It seems that utilisation of the alternative scales would make adjusting of priorities to real decision maker demands possible. Obtained results would therefore correspond better to 
actual decision maker's expectations. On the other hand, concurrent application of different prioritisation methods would deliver more justified priorities.

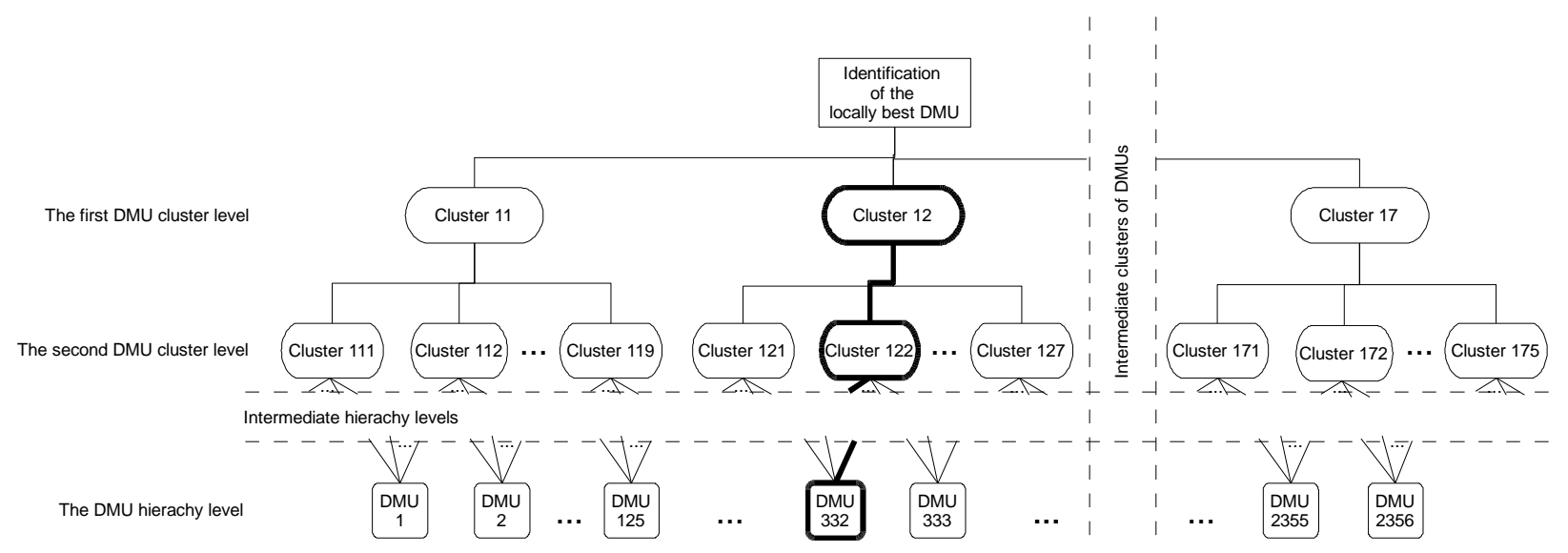

Figure 2. Structure of hierarchy of clusters and DMUs and a course of a local priority estimation

It is obvious that application some initial intangible feature measurement is scale is required for clustering of DMUs. Utilisation of any ordinal scale seem to be sufficient with this regard. Any cluster analysis or discriminant analys is tool can be applied to identif y cluster components at any level of cluster hierarchy. However, one can also use other procedures for identification of initial clusters even without a need for application of an apparent measurement scale. For example, multi-stage approach can be helpful with this regard. It is based on subjective progressive identification of groups of similar DMUs. The identification would stop after appropriate number of DMU clusters of appropriate cardinality is obtained.

\subsection{Input data for intangibility-aware prioritisation}

Successful application of the approach for prioritisation of DMUs due to intangible features requires a lot of input data. It seems a serious drawback even if serious reductions in number of really unique DMUs due to repetitive feature value are expected in practice.

There are, however, several possible ways to cope with this problem. For example, one can use a severely limited initial measurement scale to obta in small number of DMU clusters. Lower-level clusters can be then identified using scale gradual scale extension. The second possible way deals with reduction of number of compared objects thanks to providing reliable representation of a whole DMU population. The representative DMUs express whole clusters of DMUs. Evaluation of representative DMUs therefore delivers priorities for a whole cluster. There are different approaches available with regard to representative selection (Kolenda, 2006). The DMU representatives can be applied together with gradual scale extension.

Application artificial intelligence tools to reduce effort with regard to input data preparation. For example, trained artificial neural networks could be very helpful to provide numerous justified judgements pertaining to numerous importance relations between objects compared in a pair-wise manner. Moreover, application of suitable teaching data would even enable imitation habits of particular experts. Application of the networks seems therefore a very interesting urgent topic with regard to AHP/ANP utilisation practice. 


\subsection{Application of the approach}

Initial utilisation of the approach delivered interesting results. It was, however, devoted to a medium-sized decision problem. Only application of the approach to larger problems seems capable of delivering outcomes which would make evaluation of a thorough usability of the approach possible.

Preparation to such application are underway as they require a considerable amount of data.

\section{Conclusions}

Presented approach comprises a vital extension to AHP/ANP-based analysis in the case of numerous decision making alternatives. Its main advantage comes from successful coupling of reliable intangibility addressing with decision making aimed at numerous DMUs.

Outcomes of initial analysis are encouraging. Application of further research is, however, required to resolve the problems which accompany proposed approach and to further extend its idea.

\section{REFERENCES}

Dytczak, M., \& Szklennik, N. (2011). Principles and applications of AHP/ANP-based multiple MCDA methods approach. [In:] ISAHP 2011. Proceedings, Sorrento, 15-18 June 2011.

Kolenda, M. (2006): Numerical Taxonomy. Classification, Ranking and Analysis of Multivariable Objects. Akademia Ekonomiczna we Wrocławiu. Wrocław 2006.

Miller, G.A. (1958). The Magical Number Seven, Plus or Minus Two: Some Limits on Our Capacity for Processing Information. The Psychological Review, vol. 63, pp. 81-97.

Saaty T.L., \& Ozdemir M.S. (2003). Why the magic number seven plus or minus two. Mathematical and Computer Modelling, 38(3 4):233 244. 